\title{
An optimized rubber sheet model for normalization phase of IRIS recognition
}

\author{
Selvamuthukumaran.S1, Ramkumar. $\mathbf{T}^{2}$, Shantharajah $\mathbf{S P}^{3}$ \\ ${ }^{1}$ Faculty of Computer Applications, AVC College of Engineering, India \\ ${ }^{2,3}$ School of Information Technology \& Engineering, VIT University, India
}

\begin{tabular}{|c|c|}
\hline Article Info & ABSTRACT \\
\hline Article history: & Iris recognition is a promising biometric authentication approach and it is a \\
\hline Received Jan 23, 2020 & $\begin{array}{l}\text { very active topic in both research and realistic applications because the pattern } \\
\text { of the human iris differs from person to person, even between twins. In this }\end{array}$ \\
\hline Revised May 27, 2020 & paper, an optimized iris normalization method for the conversion of segmented \\
\hline Accepted Jun 16, 2020 & $\begin{array}{l}\text { image into normalized form has been proposed. The existing methods are } \\
\text { converting the Cartesian coordinates of the segmented image into polar }\end{array}$ \\
\hline Keywords: & $\begin{array}{l}\text { coordinates. To get more accuracy, the proposed method is using an optimized } \\
\text { rubber sheet model which converts the polar coordinates into spherical }\end{array}$ \\
\hline $\begin{array}{l}\text { Biometrics } \\
\text { Histogram equalization } \\
\text { IRIS normalization }\end{array}$ & $\begin{array}{l}\text { coordinates followed by localized histogram equalization. The experimental } \\
\text { result shows the proposed method scores an encouraging performance with } \\
\text { respect to accuracy. }\end{array}$ \\
\hline
\end{tabular}

This is an open access article under the CC BY-SA license.

Spherical coordinates

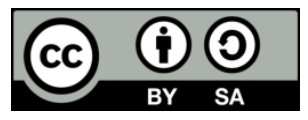

\section{Corresponding Author:}

Selvamuthukumaran.S,

Faculty of Computer Applications,

AVC College of Engineering, India.

Email:smksmk@gmail.com

\section{INTRODUCTION}

The progressive digital society demands secure identification techniques for the development of biometric system in diverse fields [1]. The iris biometric systems are becoming commonly implemented as one of the best ways to certainly identify people [2]. The iris is an outwardly and colourful organ close the pupil of the eye [3]. Figure 1 shown the structure of the eye, which demonstrates the exact position of the iris and its surrounding things. The property of the iris guarantees that even equal twins have uncorrelated iris details. Thus, the exclusivity of every iris, including the couple possessed by single individual, parallels the exclusivity of every fingerprint regardless of whether there is a common genome [4,5]. The iris involves of several irregular small blocks similar to freckles, stripes, furrows, coronas, etc. In addition, the texture divisions in the iris are arbitrary. The separate merits of the iris cause its high consistency for individual identification [6]. Hence the method of iris identification becomes an exciting research point in current years [7]. The first step in iris recognition is the process of capturing the image of an eye [8]. During stage of the image acquisition, a camera is used to record iris textures. The captured image is further processed for the localization stage, where the location of the iris is carried out, followed by the segmentation phase. In segmentation, irises are separated from the eye [6]. Next to this, a normalization of the iris image is done using various noise removal techniques, and the images are stored in the binary format [9]. The process of the iris recognition system continues with the feature extraction stage, where the extractions of various features of irises are identified [10]. In verification process, matching will be done where the acquired image is compared with the images stored in the database [11]. A typical iris recognition system which includes above stages is shown in the Figure 2. 


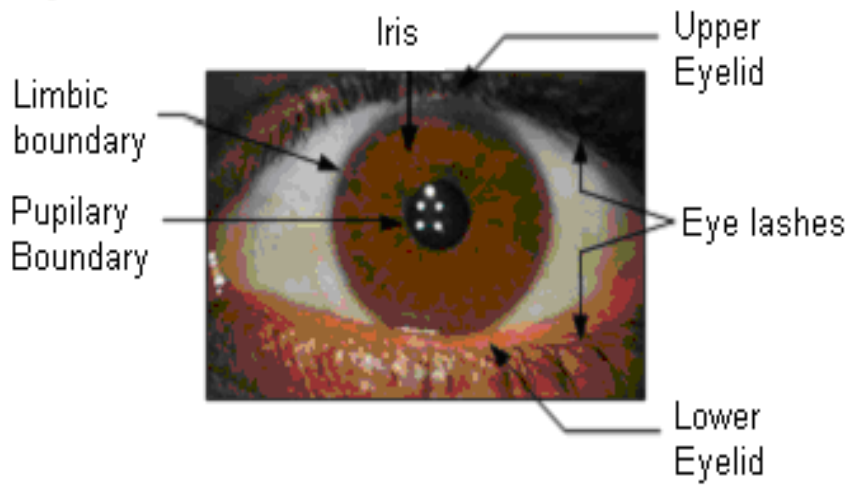

Figure 1. Structure of an eye

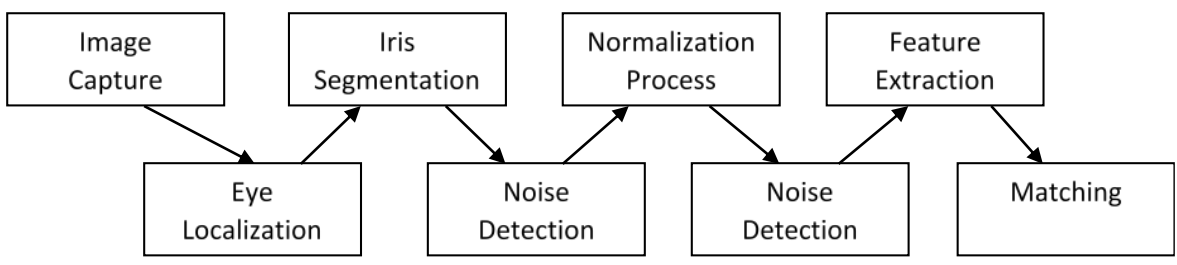

Figure2. IRIS recognition system

In the phase of normalization, segmented iris image has been prepared for the feature extraction process. Though, several of algorithms $[1,5,11]$ are available for the normalization phase of the Iris recognition, Daughman's [12] and Wilde's [13] algorithms are significant. To compensate the differences in the magnitude of the pupil, Daughman [12] proposed a rubber sheet model for normalization. In this way, some of the irises still may be sealed by eyelids or eyelashes, even when the inner and outer boundaries of the iris are reached. Wildes' system is a patent of the iris recognition system which employes the gradient-based Hough transform to choose the two circular boundaries of an iris. It has big computational charge, since it examinates among all of the possible candidates. Besides, the algorithm's accuracy significantly deteriorates while dealing with the noisy data. The above two normalization techniques transform Cartesian coordinates in to polar coordinates for un-wrapping the iris texture into a fixed size rectangular block. In polar coordinates, the distance and angular position have extremely affected iris images with respect to the camera. Additionally, illumination has a direct influence on the pupil size and sources non-linear distinctions of the iris patterns [14].

Moreover, the pupil boundary and limbus boundary are usually two non-concentric contours. The non-concentric condition leads to different choices of reference points for transforming an iris into polar coordinates, which is not suitable for iris images with more noise. Hence, it is necessary to adopt an optimization technique for transforming the iris image to compensate these variations. In this paper, we have proposed an optimized normalization technique based on rubber sheet model which converts Cartesian coordinates into spherical coordinates instead of polar coordinates and perform localized histogram equalization for further accuracy. The rest of the paper has been organized as follows; in section 2, the improvised model for iris normalization has been illustrated with the inclusive steps. The experimental investigations of the proposed work with the help of CASIA database [15] is explained in section 3, where concluding remarks and feature research directions are summarized in section 4 .

\section{AN OPTIMIZED RUBBER SHEET MODEL FOR IRIS NORMALIZATION}

The normalization stage aims to gain invariance to size, position and pupil dilatation in the segmented iris region. Most of the methods use Daughman's [12] rubber sheet model considers the possibility of pupil dilation and the appearance of different sizes in different images. For this purpose, the coordinate system is altered by un-wrapping the iris and plotting all the points within the edge of the iris into their polar coordinates. The Cartesian to polar transformation is defined for the continuous form of images. However, in the discrete form, the transformation encounters problems in which the polar samples do not entirely match the Cartesian samples which results in excessive interpolation and in some cases loss of information would be the result. The proposed method is the improvised method of Daughman's rubber sheet model and it improves the accuracy

An optimized rubber sheet model for normalization phase of IRIS recognition... (Selvamuthukumaran.S) 
by advocating a two-step process. At First, the coordinate system of the Iris region is converted from Cartesian into the spherical. Then, the remapping process is done for the normalization. Localized histogram equalization is applied on the iris region for further normalization. The various inclusive stages of the proposed work are illustrated in the Figure 3.

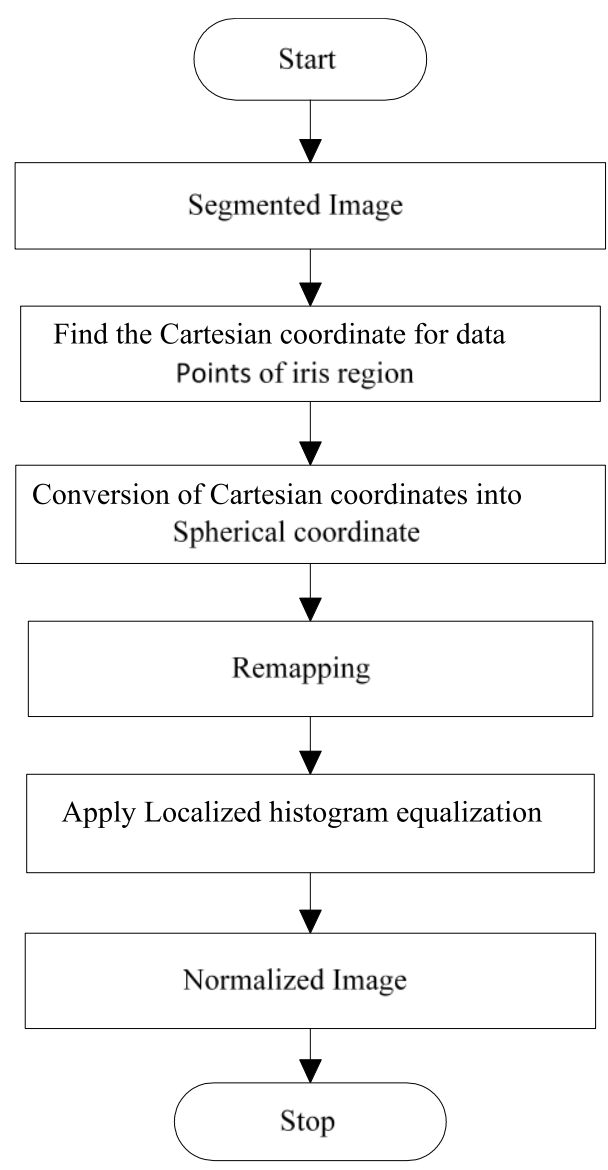

Figure3. Flowchart of proposed work

\subsection{Conversion of cartesian coordinates into spherical coordinates}

In the spherical coordinate system, the position of a point is specified by three coordinates; $(r, \theta, \varphi)$ where ' $r$ ' is the radial distance from a fixed origin, ' $\theta$ ' is the elevation angle of that point from a plane, and ' $\varphi$ ' is the azimuth angle of its orthogonal projection on that plane from a fixed direction. The elevation angle is often replaced by the inclination angle measured from the azimuth; the direction perpendicular to the reference plane. The radial distance is also named the radius or radial coordinate and the inclination is named as colatitude. The coordinate conversion is represented in Figure 4. During co-ordinate conversion, the centre of the pupil is considered as location point and the radial vector circle has been considered in the Iris region. The coordinate conversion is performed to obtain the invariance of iris size, position and different degrees of pupil dilation. It produces the iris regions, which have the same constant dimensions, with the intention that two images of the same iris should have characteristic features under different conditions at the same spatial place. The proposed technique is useful in bringing iris images into a standard fixed resolution, which simplifies the feature extraction process. In the adjusted rubber sheet model, the centre of the pupil is used as the reference point; and the radial and azimuth vectors cross the iris region. The spherical coordinates, $(r, \theta, \varphi)$ of a point can be obtained from its Cartesian coordinates, $(x, y, z)$ by the following equations. 

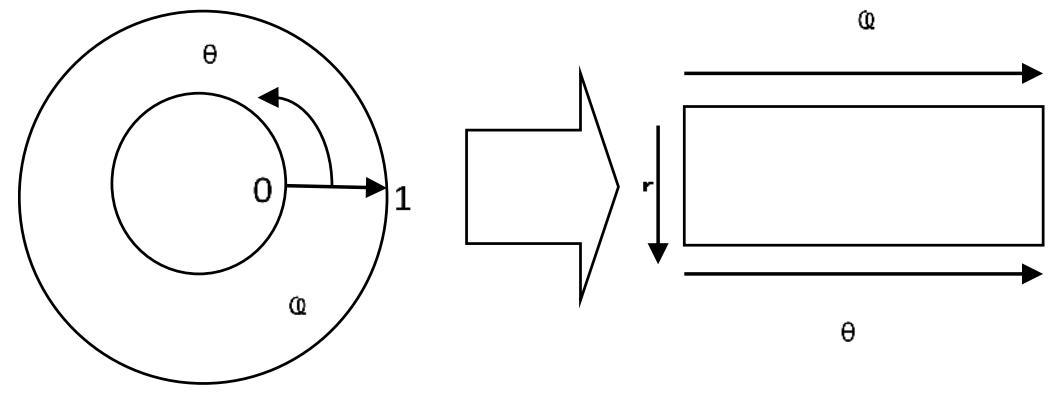

Figure4 Coordinate conversion

$$
\begin{aligned}
& r=\sqrt{x^{2}+y^{2}+z^{2}} \\
& \varphi=\operatorname{atan}(y, x) \\
& \theta=\arccos \left|\frac{x}{\sqrt{x^{2}+y^{2}}}\right|
\end{aligned}
$$

Where $\operatorname{atan}(y, x)$ is a variant of the arctangent function that returns the angle from the $x$-axis to the vector $(x, y)$ in the full range $(-\pi, \pi)$. The formulas assume that the two systems have the origin, then the spherical reference plane is the Cartesian ' $x-y^{\prime}$ plan ' $\theta$ ' is the inclination from the ' $z$ ' direction and the azimuth angles are measured using the coordinates. After converting the data points of the iris region into spherical coordinates, remapping has been done to rescale the points on the angle around the inner and outer boundaries. The remapping of the iris region from ' $(x, y, z)^{\prime}$, the Cartesian coordinates to the normalised spherical representation can be modelled as in the following equation.

$$
I(x(r, \theta, \varphi), y(r, \theta, \varphi), z(r, \theta, \varphi))=I(r, \theta, \varphi)
$$

Where ' $I(x, y, z)$ ' is the iris region image, ' $(\mathrm{x}, \mathrm{y}, \mathrm{z})$ ' are the original Cartesian coordinates, and ' $(r, \theta, \varphi)^{\prime}$ are the corresponding normalized spherical coordinates. It also represents the coordinates of the pupil and iris boundaries along the ' $\theta$ ' direction and furnished in the following equations.

$$
\begin{aligned}
& I_{n}(x, y, z)=I_{O}(x, y, z) \\
& x=x_{p}(\theta, \varphi)+\left(x_{i}(\theta, \varphi)-x_{p}(\theta, \varphi)\right) \frac{1}{m} \\
& y=y_{p}(\theta, \varphi)+\left(y_{i}(\theta, \varphi)-y_{p}(\theta, \varphi)\right) \frac{1}{m} \\
& z=z_{p}(\theta, \varphi)+\left(z_{i}(\theta, \varphi)-z_{p}(\theta, \varphi)\right) \frac{1}{m}
\end{aligned}
$$

where $\theta=2 \pi \frac{x}{N}$,

Here ' $I_{n}{ }^{\prime}$ is the ' $M \times N^{\prime}$ normalized image and ' $x_{p}(\theta, \varphi), y_{p}(\theta, \varphi), z_{p}(\theta, \varphi)^{\prime} \quad$ and ' $x_{i}(\theta, \varphi), y_{i}(\theta, \varphi), z_{i}(\theta, \varphi)$ ' are the coordinates of the inner and outer boundary points. ' $\theta$ ' is the inclination from the ' $Z$ ' direction in the original image, ' $I_{O}$ '. This process ends with the normalised Iris image, which is further forwarded for histogram equalization with the view of getting better accuracy.

\subsection{Localized histogram equalization}

The normalized image, resultant of the previous phase may have low intensity contrast and improper brightness due to the position of the light sources and other issues. In such situations, to achieve more accurate results, histogram equalization has been chosen. This method is a contrast enhancement technique with the objective to obtain a new enhanced iris image with a uniform histogram. It distributes the histogram of the pixel levels in a uniform manner without any loss. It has been implemented for improving the look of a noisy image by amplifies the global contrast of the iris image. The histogram, ' $H[x]$ ' contains pixels with the value, 
' $x$ '. The cumulative density function of the histogram ' $c d f[x]$ ' contains the number of pixels with the value, ' $x$ ' or less is given by the following equation.

$$
(c d f[x])=H[0]+H[1]+H[2]+\cdots+H[x]
$$

It should be applied to each pixel in the Iris image and replace the existing value with the calculated value. The histogram of a digital image in the range of $[0, K-1]$ is a discrete function as given in the following equation.

$$
p\left(r_{k}\right)=\frac{n_{k}}{n}
$$

Where ' $r_{k}$ ' is the $\mathrm{k}^{\text {th }}$ gray level, is the number of pixel in the image with that gram level, ' $\mathrm{n}$ ' is the total number of pixels in the image, and $\mathrm{k}=0,1,2, \ldots, \mathrm{K}-1 . p\left(r_{k}\right)$ Gives the probability of occurrence level, $r_{k}$.

Consider the following sample original entire eye shown in the following Figure 5.

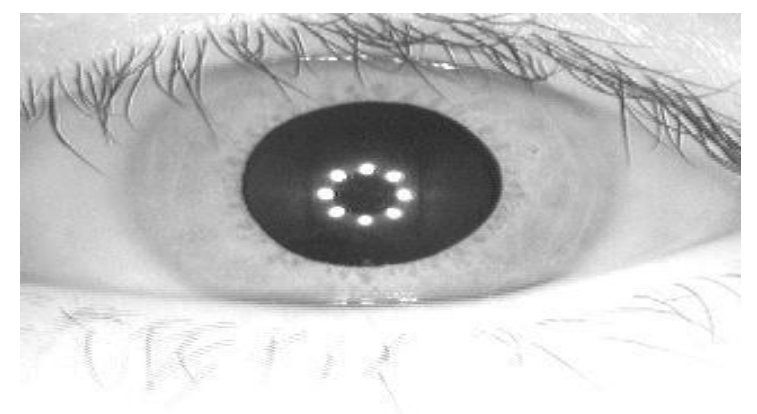

Figure 5. Original image before localized histogram equalization

The contrast of the iris image is amplified, mainly when the used data of the iris image is represented by near contrast values. The intensity would be better spread on the histogram, through this fine-tuning. It changes the areas of lower contrast to rise a higher contrast without distressing the overall precision of the irises. The feature of interest in the image may require enrichment locally. Histogram equalization reaches this by efficiently spreading out the most frequent intensity values. By using this method, the histogram of the resultant image is as flat as possible. The resultant histogram equalized image is shown in the Figure 6.

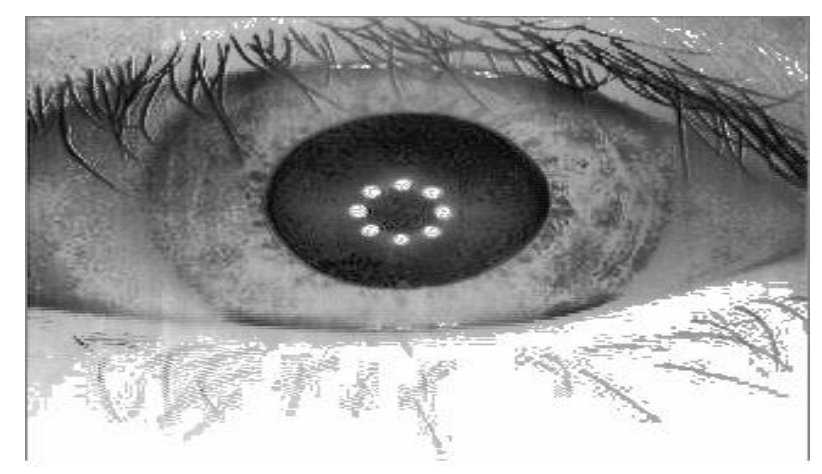

Figure 6. Image after the localized histogram equalization

It consists of applying localized histogram equalization independently to capture the iris region in the eye image since most small regions are very self-similar. If the image is made up of discrete regions, most small regions lie entirely within one or the other region. This method attempts to equalize the number of pixels which tend to flatten and raise an image's histogram. Vertical equalization uses only a single column of pixels into the equalization process, whereas horizontal equalization uses a single row of pixels. It modifies the pixel 
intensities for a better appearance of the Irises. The proposed method improves the visual appearance [16] of an image.

\section{EXPERIMENTAL STUDY}

To achieve a size-invariant sampling of the valid iris pixel points, we have applied the optimized rubber sheet model to map the sampled iris pixels from the Cartesian coordinates into the normalized spherical coordinates. To effectively implement our proposal, the iris image database CASIA Database Version 3.0 (CASIA-IrisV3) released by the Centre for Biometrics and Security Research of National Laboratory of Pattern Recognition, Chinese Academy of Sciences has been used [15]. The pupil is smaller in the image; however, the normalisation process can able to rescale the iris region, so that it has a constant dimension. In our experimental study, the rectangular representation is constructed from 12,000 data points in the iris region. The remapping is done using the spherical coordinates. The Iris shown in Figure 7 is the segmented image of S1046R04.

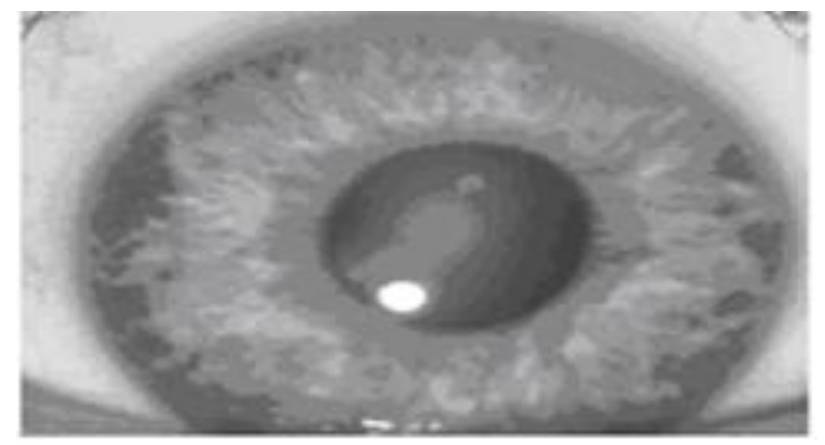

Figure 7. Segmented Iris image of S1046R04

After the successful conversion of the Cartesian coordinate into the spherical coordinate system, the normalised Iris is shown in the following figure. Comparing the results from the below Figure 8 and Figure 9, it has been observed that the distribution is shifted towards the higher values, while the peak at the minimum intensity remains after the localized histogram equalisation. It recovered some of the apparently lost contrast in an image, by remapping the brightness values in such a way as to equalize, or more evenly distribute its brightness values. The histogram of the normalised iris of Figure 8 is shown in Figure 10. The unwrapped flat iris has a low contrast. This iris is enhanced by applying localized histogram equalization. The resultant image is shown in the following figure. The histogram of the normalised iris of Figure 9 after the localized histogram equalization is shown in Figure 11.

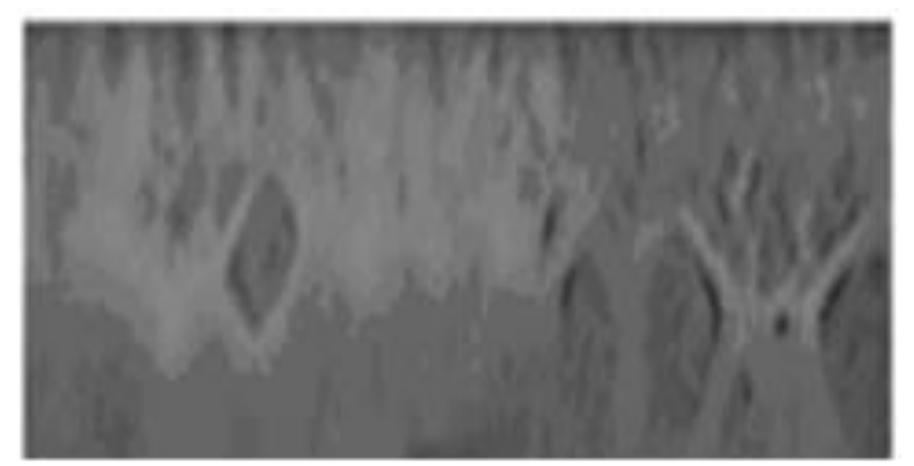

Figure 8. Normalized IRIS 


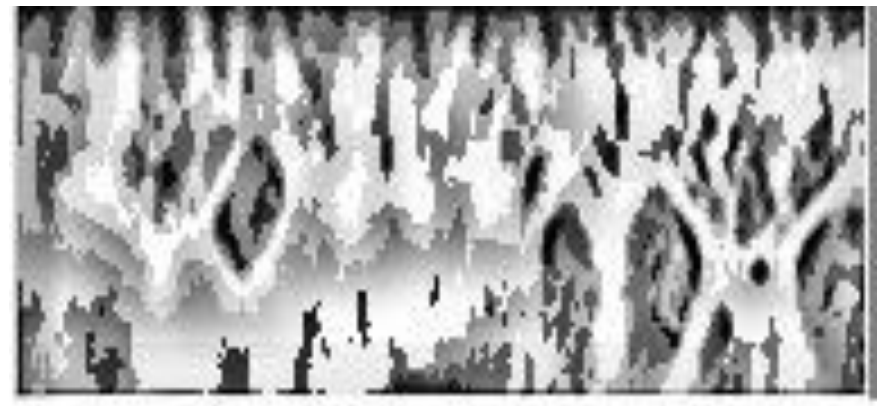

Figure 9. Normalized IRIS after histogram equalization

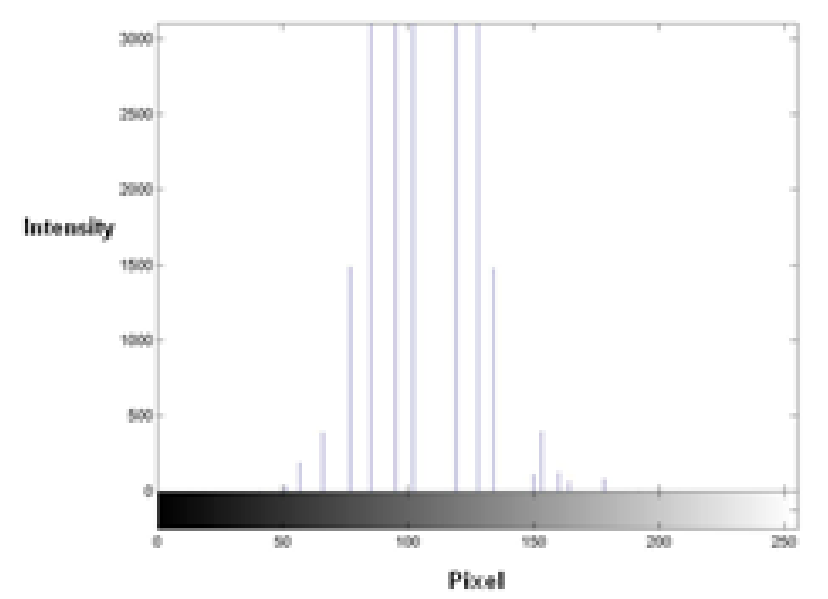

Figure 10. Histogram of normalized IRIS after conversion into spherical coordinates

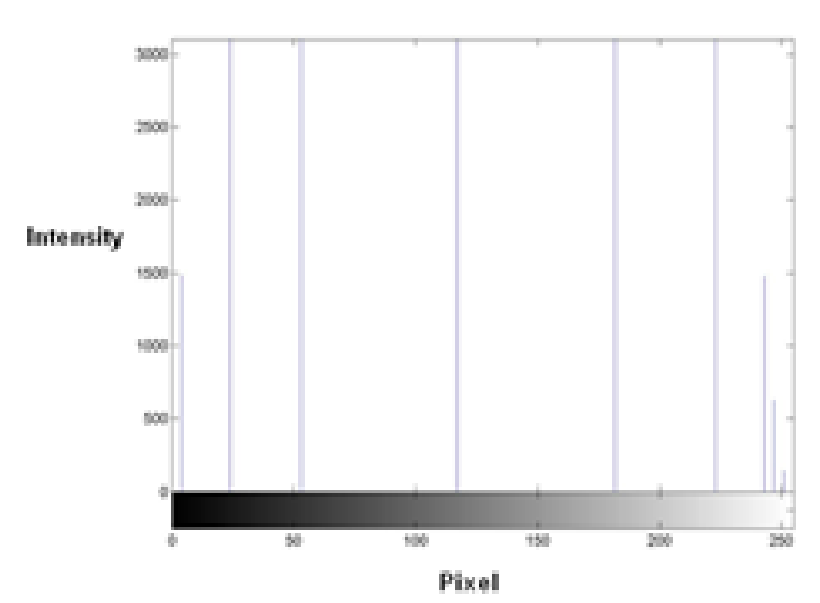

Figure 11. Histogram of the normalized IRIS after applying the localized histogram equalization

By comparing the Histograms (Figure 10 and Figure 11), it has been observed that the intensity variations are equally normalised after the localized histogram equalisation. Also output of the proposed method for some of the CASIA images are shown in the Table 1, As a result, noises in the flat regions and ring artefacts at the edges are normalised, and now the irises are ready for further processing. 


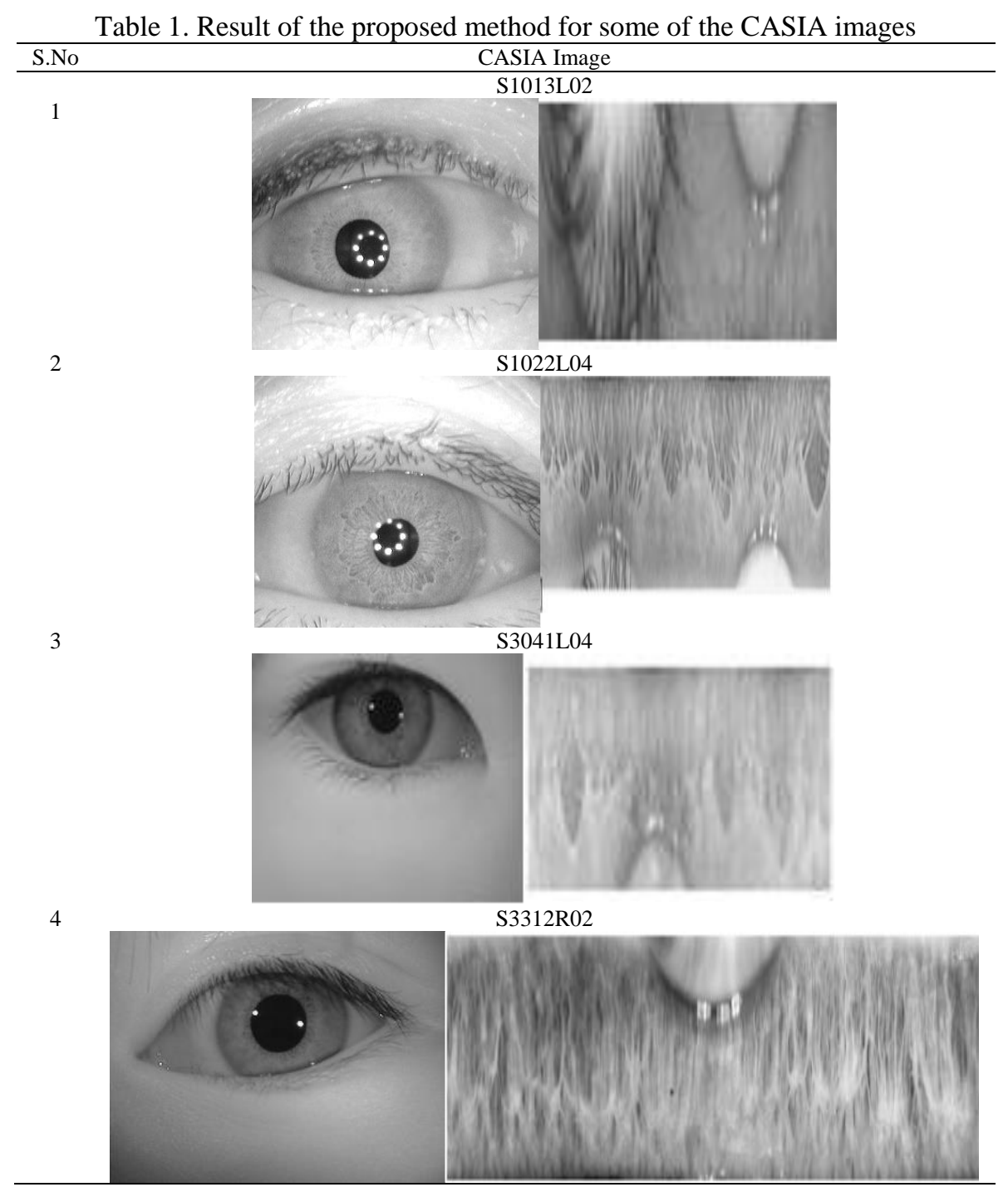

The proposed segmentation method normalized the iris region in 658 images out of 670 images, which corresponds to a success rate of $98 \%$. The success rate of the proposed method is compared with those of the previous methods Daughman's Rubber sheet model and Wild's Image registration. The success rate of the different normalization methods is shown in Table 2.

Table 2. Success rate of the normalization methods

\begin{tabular}{lcc}
\hline Normalization Method & No of irises (out of 670) & Success Rate percentage \\
\hline Optimized rubber sheet model using spherical coordinates & 658 & 98 \\
Rubber sheet model & 638 & 95 \\
Wild's Image registration & 597 & 89 \\
\hline
\end{tabular}

Table 2 shows the strength of the proposed method which works better than the existing methods since $98 \%$ of accuracy for the irises and the graphical representation of comparison is also provided in the Figure 12. 


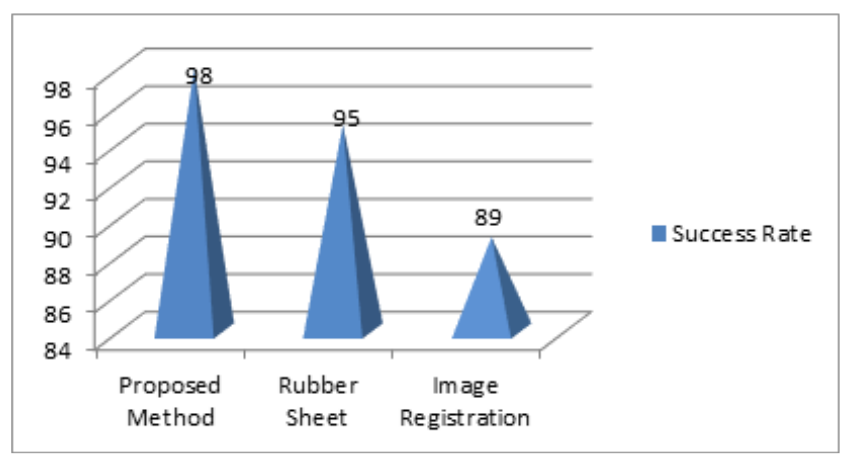

Figure12. Results of various normalization methods

\section{CONCLUSION}

In a progressively digital society, the role played by the iris recognition system is a vital one. The trends and traits made in security-based technologies demand various new algorithms for quality images. In this paper, an optimization technique for the normalization phase has been implemented which performs the conversion of the Cartesian coordinates into spherical coordinates and uses localized histogram equalization. The results obtained reveal the exact normalization of irises for the noisy images. As a future work, we put forth certain improvements in noise removal methods, Iris region resize methods towards the better feature extraction of iris images,

\section{REFERENCES}

[1] N. Mojtaba, G. Sedigheh. "IRIS recognition based on using ridgelet and curvelet transform.” International Journal of Signal Processing, Image Processing and Pattern Recognition. vol. 4, no. 2, pp. 7-18, 2011.

[2] R.M. Da Costa, A. Gonzaga. "Dynamic features for Iris recognition." IEEE Transactions on Systems, Man and Cybernetics. vol. 42, no. 4, pp. 1072-1082, 2012.

[3] A.D. Rahulkar, R.S. Holambe. "Half-Iris feature extraction and recognition using a new class of biorthogonal Triplet Half-band filter bank and flexible k-out-of-n: A Postclassifier." IEEE Transactions on Information Forensics and Security. vol. 7, no. 1, pp. 230-240, 2012.

[4] G. Yang, S. Pang, Y. Yin, Y. Li, X. Li. "SIFT based iris recognition with normalization and enhancement." International Journal of Machine Learning and Cybernetics. vol. 4, no. 4, pp. 401-407, 2012.

[5] H. Proença. "IRIS Biometrics: indexing and retrieving heavily degraded data." IEEE Transactions on Information Forensics and Security. vol. 8, no. 12, pp. 1975-1985, 2013.

[6] F.A. Santos, F.A. Faria, L.A. Villas. "IRIS recognition based on local binary descriptors." IEEE Latin America Transactions. vol. 13, no. 8, pp. $2770-2775,2015$.

[7] S. Selvamuthukumaran, S. Hariharan, T. Ramkumar, Investigation on Iris recognition system adopting cryptographic techniques, The International Arab Journal of Information Technology. 12 (1) (2015) 1-8.

[8] R. Himanshu, Y. Anamika, Iris recognition using combined support vector machine and hamming distance approach. Expert Systems with Applications. 41 (2) (2014) 588-593.

[9] Z. Peng, H. Wang, J. Wu, J. Li. "An improved Daugman method for Iris recognition." Wuhan University Journal of Natural Sciences. vol. 20, no. 3, pp. 229-234, 2015.

[10] [A. Bansal, R. Agarwal, R.K. Sharma. "Statistical feature extraction-based iris recognition system." Sadhana, Indian Academy of Sciences. vol. 41, no. 5, pp. 507-518, 2016.

[11] I. Hamouchene, S. Aouat. "Efficient approach for Iris recognition." Signal Image and Video Processing. vol. 10, no. 7, pp. 1361-1367, 2016.

[12] J.G. Daugman, "How iris recognition works." IEEE Transactions on Circuits and Systems for Video Technology. vol. 14, no. 1, pp. 21-30, 2004.

[13] R.P. Wildes, J.C. Asmuth, G.L. Green, S.C. Hsu, R.J. Kolczynski, J.R. Matey, S.E. McBride. “A machine vision system for Iris recognition." Machine Vision and Applications, vol. 9, pp.1-8, 1996.

[14] H.S. Ali, A.I. Ismail, F.A. Farag, F.E. Abd El-Samie. "Speeded up robust features for efficient Iris recognition." Signal Image and Video Processing. vol. 10, no. 8, pp. 1385-1391, 2016.

[15] CASIA iris image database. The National Laboratory of Pattern Recognition (NLPR). Institute of Automation, Chinese Academy of Sciences (CASIA-IrisV1).

[16] Y. Chen, J. Yang, C. Wang, N. Liu. "Multimodal biometrics recognition based on local fusion visual features and variational bayesian extreme learning machine." Expert Systems with Applications. vol. 64, pp. 93-103, 2016. 